\title{
Efeitos da Pandemia do COVID-19 na Transformação Digital de Pequenos Negócios
}

\author{
Effects of COVID-19 Pandemic on Digital Transformation of Small Businesses
}

\author{
Djalma Silva Guimarães Júnior ${ }^{1,2}$ (B) orcid.org/0000-0002-4539-8492 \\ Ademir Macedo Nascimento1,2 (D) orcid.org/0000-0001-5678-2740 \\ Gerlane Pereira de Albuquerque Rodrigues ${ }^{\mathbf{1 , 2}}$ (B) orcid.org/0000-0003-0233-3127 \\ Letícia Oliveira Claizoni dos Santos ${ }^{1,2,3}$ (D) orcid.org/0000-0002-6917-9091
}

\author{
${ }^{1}$ Faculdade de Ciência da Administração e Direito de Pernambuco, Universidade de Pernambuco, Recife, Brasil, \\ 2 Mestrado em Gestão do Desenvolvimento Local Sustentável, Universidade de Pernambuco, Recife, Brasil, \\ ${ }^{3}$ Universidade Federal Rural de Pernambuco, Recife, Brasil, \\ E-mail do autor principal: Djalma Silva Guimarães Júnior djalma.quimaraes@upe.br
}

\section{Resumo}

A pandemia de COVID-19 tem ceifado milhares de vidas ao redor do mundo e provocado inúmeras dificuldades para o desenvolvimento das atividades econômicas. Nesse contexto, as atividades de varejo foram especialmente transformadas, uma vez que algumas das recomendações base da Organização Mundial de Saúde foram quarentena e isolamento social, medidas que impossibilitam o acesso de consumidores aos estabelecimentos, além de afetar a capacidade de produção. Esta pesquisa objetiva investigar iniciativas de uso de ferramentas digitais em processos realizadas por micro e pequenas empresas (divulgação, comercialização, pagamento e distribuição), no contexto da pandemia do COVID19. Para isso, foi realizado um estudo exploratório no qual foram contatados por telefone 50 proprietários de pequenos estabelecimentos que não possuíam nenhuma estratégia digital antes da pandemia. Através da análise dos dados, foi possível constatar uma incipiente transformação digital forçada, que pode ser caracterizada por divulgação através de redes sociais, negociação através de aplicativos de troca de mensagens, pagamento prioritariamente eletrônico, e entregas através de serviços de aplicativos, por meio de motos e bicicletas. A combinação de diferentes tecnologias tem contribuído para a sobrevivência dos pequenos negócios e o abastecimento da sociedade em tempos de pandemia.

Palavras-Chave: Digitalização, Micro e pequenas empresas, Estratégia de negócios, Aplicativos.

\section{Abstract}

The COVID-19 pandemic has claimed thousands of lives around the world and caused various difficulties for the development of economic activities. In this context, retail activities were especially transformed since some of the basic recommendations of the World Health Organization were quarantine and social isolation. These measures make it impossible for consumers to access establishments, also affecting production capacity. This research aims to investigate initiatives for the use of digital tools in processes carried out by micro and small companies (disclosure, commercialization, payment and distribution), in the context of the COVID-19 pandemic. For this, an exploratory study was conducted with 50 owners of small business, who had no digital strategy before the pandemic. They were contacted by telephone. Through data analysis, it was possible to observe a forced digital transformation in a beginning stage, which can be characterized by disclosure through social media, negotiation through messaging applications, primarily electronic payment, and deliveries through application services, by motorcycles and bicycles. The combination of different technologies has contributed to the survival of small businesses and the supply of society in times of pandemic.

Key-words: Digitalization, Micro and small businesses, Business strategy, Applications. 


\section{Introdução}

O surgimento e a propagação do COVID-19 têm levado a morte de centenas de milhares de pessoas ao redor do mundo, e gerado sobrecarga na operação dos sistemas de saúde, em decorrência da expansão exponencial da demanda por serviços de saúde. À medida que o vírus se alastrou pelo mundo, medidas de contenção foram endurecidas, sobretudo a partir do exemplo chinês, na província de Hubei, onde foram adotadas medidas rígidas para redução do contágio. De acordo com OLIVEIRA et al [1], como recomendação da Organização Mundial de saúde [2] [3], para alcançar a diminuição do ritmo de contágio, e a consequente diluição da demanda por leitos de unidade intensiva ao longo do tempo, algumas das principais medidas adotadas foram: quarentena, isolamento social e o fechamento de todas as atividades que não são considerados essenciais.

Por conseguinte, a pandemia também trouxe um efeito extremamente nocivo para a economia dos países. Segundo previsão do Fundo Monetário Internacional [4], o PIB mundial deve retrair 3\% em 2020, e o brasileiro, 5,3\%. Mesmo antes da crise, o Brasil vivenciava uma tímida recuperação da economia após uma profunda recessão, agravada por um déficit fiscal persistente desde 2014, de acordo com o Banco Central (Bacen) [5]. Outro agregado macroeconômico afetado foi o emprego. Estima-se que o percentual de desempregados amplie de $11,6 \%$ para $16,1 \%$, que representa 0 incremento de 5 milhões de pessoas na massa de desempregados no país, conforme previsão da FGV [6].

Os efeitos adversos da pandemia do COVID-19 têm levado os países a adotar medidas de estímulo à economia e proteção social. O Governo dos EUA [7] anunciou um programa para injetar US $\$ 2$ trilhões na economia, destinados ao apoio a desempregados e empresas; a União Europeia, através da European Comission [8], por sua vez, aprovou um investimento anticrise que chega a US $\$ 1,08$ trilhão distribuídos na forma de garantias de créditos para empresas e auxílio para vulneráveis. O Governo Federal do Brasil [9] também apresentou um programa de enfrentamento à crise, que direcionou US $\$ 28,2$ bilhões à população mais vulnerável e à manutenção de emprego.

Nesse contexto, as empresas de menor porte têm enfrentado inúmeros desafios, como queda ou interrupção da operação, retração da demanda, custos persistentes mesmo com a paralisação da operação, expectativas negativas dos consumidores e 2 dificuldade de acesso a crédito. O Serviço Brasileiro de Apoio às Micro e Pequenas Empresas (SEBRAE) estima que 602 mil micro e pequenas empresas tendem a fechar no Brasil em virtude dos efeitos da pandemia do COVID-19, conforme publicação da CNN [10]. Por essa razão, empreendedores individuais, micro e pequenas empresas necessitam de mecanismos de apoio. Uma iniciativa é o Programa Emergencial de Manutenção do Emprego e Renda (MP 963/2020) [11], que permite a suspensão de contratos de trabalho e redução da jornada com compensação dos salários por parte do governo federal, além da criação de uma linha de crédito emergencial para financiar o salário durante dois meses.

Mesmo antes de qualquer auxílio governamental, muitos negócios - sobretudo do varejo - buscaram formas de reinventar seu modelo de negócios, a fim de continuar em operação. E o auxílio das tecnologias digitais tem possibilitado a continuidade da comercialização e o consumo de bens e serviços. Esta pesquisa pretende investigar a ocorrência da digitalização de processos de negócios em micro e pequenas empresas e empreendedores individuais, decorrente dos impactos da pandemia.

O trabalho está organizado da seguinte maneira: nesta seção foi apresentado o contexto e problemática da pesquisa; na próxima seção é desenvolvido o fundamento teórico basilar da pesquisa, o contexto dos pequenos negócios no país, comércio eletrônico e transformação digital, e problemas de distribuição da última milha; na terceira seção, é apresentado o procedimento metodológico utilizado na pesquisa; por fim, nas seções seguintes são relatados os resultados e discutidas as principais conclusões.

Cabe salientar, o aspecto de originalidade e ineditismo presente neste estudo e que se reflete nos resultados da pesquisa. Tecnologias disponíveis e que até então eram usadas como entretenimento começam a ser utilizadas de forma variável envolvendo o aspecto profissional. Nos resultados da pesquisa fica evidenciado o predomínio do uso do aplicativo WhatsApp; maciça utilização do cartão de crédito e a utilização de serviços de entrega terceirizados fortalecem o objetivo da pesquisa através destes resultados que é evidenciar o panorama das mudanças forçadas mediante 0 surgimento da pandemia do Covid-19. 


\section{Fundamentação teórica}

O trabalho apresentado é um estudo sobre os reflexos que a pandemia do Covid-19 exerceu na utilização forçada e tempestiva dos recursos tecnológicos pelas micro e pequenas empresas com o intuito de minimizar o prejuízo em um cenário de incertezas econômicas. Os temas abordados nesta pesquisa a saber, são: a digitalização nas micro e pequenas empresas, as estratégias de negócios para fidelizar clientes neste cenário de imprevisibilidade e demais aspectos envolvidos na concretização das operações neste ambiente de reinvenção comercial.

\subsection{Panorama das micro e pequenos negócios no Brasil}

No Brasil, as Leis Complementares $n^{\circ} 123, n^{\circ} 128$ e $n^{\circ} 155$ [12] [13] [14] estabelecem um tratamento diferenciado às microempresas e empresas de pequeno porte, através de normas gerais. São classificadas como microempresas as organizações que possuem receita bruta anual de até $R \$$ $360.000,00$ e empresas de pequeno porte as que possuem receita bruta de até $R \$ 4,8$ milhões. Outra categoria considerada no mercado brasileiro é a figura do Microempreendedor Individual (MEI), definido pela Lei Complementar no 128, o qual possui um regime tributário desburocratizado, tal empreendimento deve possuir receita bruta anual de $\mathrm{R} \$ 81.000,00$.

A taxa total de empreendedorismo (TTE) no Brasil, segundo o Global Entrepreneurship Monitor Empreendedorismo no Brasil [15], 51,9 milhões de pessoas ou $38 \%$ da população do país está envolvida na criação ou manutenção de algum negócio. Tal patamar é o segundo maior da série histórica iniciada em 2002. O persistente cenário recessivo da economia nos últimos anos tem propiciado um ambiente desafiador para os empreendedores e isso se refletiu em um aumento na taxa de insucesso nos empreendimentos. O SEBRAE [16] destaca os principais motivos apontados como responsáveis pela mortandade precoce das empresas: falta de capital (19\%), encontrou outra atividade de atuação (14\%), falta de clientes (9\%), problemas particulares $(9 \%)$ e problemas de planejamento/administração ( $8 \%)$.

Durante a pandemia, uma pesquisa realizada pelo SEBRAE [17] com empreendedores individuais e proprietários de empresas micro empresas e de empresas de pequeno porte indicou que $58,9 \%$ das organizações interromperam temporariamente 0 3 funcionamento, enquanto $31 \%$ modificaram a forma de funcionamento, $6,6 \%$ continuaram funcionando da mesma maneira, e 3,5\% encerraram as atividades. Dentre o grupo de empresas que modificou o modelo de funcionamento, $41,9 \%$ passou a funcionar através de vendas online. A pesquisa ainda revelou que $73,4 \%$ das empresas estava com situação financeira razoável ou ruim, enquanto apenas $26,6 \%$ consideraram a situação das finanças boa.

O setor de alimentação fora do lar tem grande importância para a economia brasileira. O segmento é um dos que apresenta maior crescimento e já representa $34 \%$ dos gastos com alimentos dos lares brasileiros. No entanto, apenas $28 \%$ dos respondentes da pesquisa Consumer Reports on Eating Share Trends (CREST), realizada pela GS\&NPD para o Instituto Foodservice Brasil (IFB) [18], em fevereiro de 2019, relataram realizar ao menos 1 refeição preparada fora do lar em um dia. Entre os 13 países participantes da pesquisa, o Brasil é o que apresenta 0 menor resultado, o que indica um potencial crescimento para o segmento.

Diante da impossibilidade de consumo no local, muitos serviços de varejo, sobretudo 0 de alimentação, e também produtores de alimentos para venda em locais físicos (tais como bolos, chocolates etc) modificaram seus negócios, através de inovações. No segmento de alimentos e bebidas, SEBRAE [17] aponta que $43 \%$ dos pequenos negócios afirmou realizar mudanças no funcionamento. Como apresentado por GS\&NPD [18], o segmento já tem aptidão e grande potencial de crescimento para demanda de delivery, e drivethru, mas demanda uma estrutura diferenciada, maior consumo de embalagens descartáveis, processos de higienização mais rigorosos diante das incertezas de contaminação do corona vírus, além de mudanças no atendimento dos pedidos.

\subsection{Comércio Eletrônico e transformação digital}

O comércio eletrônico vem ganhando espaço em vários países ao redor do mundo nos últimos anos, sendo o Brasil o país com o maior faturamento nessa área na América Latina. Vale destacar ainda que, embora o PIB brasileiro tenha apresentado um crescimento tímido, o comércio eletrônico teve um crescimento nominal de $20 \%$ em 2017 e 18\% em 2018, de acordo com dados da EBIT-NIELSEN [19]. 
Mesmo com os números positivos do comércio eletrônico no Brasil, poucos negócios vêm se preparando para a transformação digital, uma vez que esse processo exige mudanças de modelos de negócio para sua efetivação, conforme apontam Okano, Simões, e Langhi [20].

Ademais, devido ao fechamento do comércio para conter a proliferação da Covid-19 no primeiro semestre de 2020, a venda por meios digitais se apresenta como uma das principais soluções para evitar a falência de empresas e o aumento do desemprego. Dessa forma, negócios que estavam num estágio avançado de transformação digital puderam se adaptar melhor a essas novas condições, enquanto que negócios tradicionais se viram forçados a se adaptar abruptamente.

Diferente do que se difunde, a transformação digital não está relacionada com tecnologia, mas sim com estratégia e maneiras de pensar. De acordo com Rogers [21], para transformar-se na era digital é necessário que o gestor atualize a sua mentalidade estratégica, muito mais que a sua estrutura de TI. Nessa linha, Schallmo, Williams e Boardman [22], destacam que a transformação digital engloba empresas e clientes em todos os segmentos da cadeia de valor agregado por meio da aplicação de novas tecnologias, exigindo habilidades que envolvem extração e troca de dados, bem como a análise e conversão desses dados em ações.

Assim, para que as pequenas empresas possam acompanhar o ritmo da transformação digital é preciso avaliar em que estágio cada negócio se encontra para identificar as estratégias de inovação mais assertivas. De acordo com Diogo et al [23], a perspectiva ampla desta transformação aliada ao gerenciamento do conhecimento eleva a competitividade das empresas garantindo a permanência e consolidação dos negócios.

No que concerne ao marketing digital, os sites de buscas e as redes sociais (em especial o Facebook e o Instagram) são apontados como os principais motivadores de compra no Brasil. Ainda sobre este aspecto, o relatório da EBIT-NIELSEN [19] destaca que $80 \%$ dos consumidores que compram por indicações de redes sociais ficaram satisfeitos e elogiaram a empresa. Mesmo em situações incomuns e em ambientes turbulentos, a capacidade de desenvolver ações de marketing se revela fundamental para o crescimento das vendas das organizações.
Em relação às plataformas digitais, nota-se que existem atualmente tanto soluções próprias, mais comumente desenvolvidas por grandes empresas, e soluções gerais, que buscam conectar várias empresas aos consumidores locais, atuando como mecanismos facilitadores da última milha. Dentre as soluções gerais, destacam-se principalmente as relacionadas a alimentos e bebidas, como é o caso do UberEats ${ }^{\circledR}$, James $^{\circledR}{ }^{\circledR}$, Rappi $^{\circledR}$ e Ifood $^{\circledR}$, sendo esta última uma empresa brasileira que atingiu um valor de mercado de $\mathrm{R} \$ 1$ bilhão em 2019 [24].

Já em relação à comercialização de bens duráveis, é possível destacar o marketplace da Magazine Luiza ${ }^{\circledR}$, que possui mais de 11 mil micro e pequenas empresas cadastradas, ofertando produtos na plataforma em 2019, de acordo com a EXAME [24].

Além disso, há pequenas empresas que buscam utilizar as redes sociais tanto para o marketing digital como ferramenta de gestão de pedidos. Assim, Facebook, Instagram e WhatsApp passam a atuar como centro das operações de diversos negócios durante o isolamento social.

\subsection{A última milha}

A fase final da cadeia de suprimentos, denominada de última milha, representa o transporte do produto até o ponto de venda ou ao cliente final. Esta fase é considerada um dos elementos mais críticos no processo de atendimento dos pedidos, segundo Gevaers et al. [25].

Alguns desafios para o gerenciamento da última milha são apresentados por Alenn et al. [26], elevado custo do atendimento dos pedidos, entregas gratuitas, expectativas elevadas dos clientes em relação ao prazo de entrega, janelas de horários de entrega, rastreabilidade e personalização da remessa. Janjevic e Winkenback [27], ainda apontam que problema da última milha ainda enfrenta dificuldades adicionais em áreas urbanas, tais como: nível de congestionamento, regulamentação de movimentação cada vez mais rigorosa e falta de infraestrutura logística dedicada.

A gestão da última milha nos mercados emergentes enfrenta dificuldades adicionais, que segundo Blanco e Fransoo [28] estão relacionadas a: características topológicas, má qualidade da infraestrutura de transportes, as quais muitas vezes não são acessíveis para veículos maiores ou mesmo menores, e a segurança. 
O uso de bicicletas é uma alternativa para otimização das atividades de última milha, que é investigado por Zuo et al. [29]. Segundo os autores, historicamente as malhas viárias urbanas foram concebidas para a movimentação de veículos, porém é crescente $o$ investimento das grandes cidades ao redor do mundo na ampliação na infraestrutura para o uso de bicicletas, as quais são consideradas como um conector para melhoria da acessibilidade e equidade no trânsito.

Já Kapser e Abdelrahman [30] apresentam o potencial dos veículos de entrega autônomos para a revolução das entregas na última milha de maneira mais sustentável e focada no cliente. Os autores analisaram a aceitação do uso de tais veículos na Alemanha e identificaram que o menor preço do serviço e a expectativa de desempenho são os fatores mais importantes para a aceitação do serviço.

Uma grande pressão para a melhoria do desempenho dos serviços de última milha emerge do crescimento do comércio eletrônico. A cada ano, o volume de produtos adquiridos em lojas virtuais cresce ao redor do mundo, aumentando a demanda por serviços de entrega. Para sobrepujar tais desafios, os gestores da última milha no comércio eletrônico devem buscar modelos de distribuição que apresentem um bom desempenho em várias dimensões, tais como: custo-benefício, satisfação do cliente e sustentabilidade, como destacam Janjevic e Winkenback [27]. De acordo com Ferreira [31], as inovações disruptivas impõem novos hábitos de consumo, deixando as empresas que não acompanham as mudanças, totalmente obsoletas e fora do mercado. Adicionalmente, deve-se observar aspectos de relevância para o consumidor no serviço logístico do comércio eletrônico. Nesse sentido, Guimarães Jr, Sant'Anna, Soares, Medeiros e Melo [32] identificaram que o que mais importa para o usuário do serviço são os elementos tangíveis (veículos e equipamentos), capacidade de resposta da organização a demandas dos usuários e a empatia dos prestadores de serviço.

\section{Metodologia}

Neste estudo de caráter exploratório, foi utilizado o método estatístico, a fim de analisar quantitativamente a adoção de novas estratégias de negócios. A coleta de informações acerca das estratégias de negócio de empreendedores individuais, microempresas e empresas de pequeno 5 porte foi realizada em duas etapas: I - identificação de empresas que passaram a vender seus produtos a distância após as medidas de isolamento social. Nesta etapa foram realizadas buscas em redes sociais e sites de busca; e II - coleta de dados sobre divulgação de produtos, negociação e venda, pagamento e entrega com os responsáveis pelas organizações.

Os descritores utilizados na primeira etapa foram: supermercados, mercados, hortifruti, quitandas, frigoríficos, padarias, panificadoras, delivery, entrega em casa. Os segmentos para os quais as buscas foram direcionadas não faziam uso de tecnologias digitais antes da pandemia. Foi considerada como área de abrangência a Região Metropolitana do Recife no Estado de Pernambuco, Brasil.

Foram considerados anúncios iniciados apenas após o começo período de isolamento social no estado de Pernambuco, Brasil em 17 de março de 2020. Após a identificação dos anúncios é feito o contato com a empresa para coleta das seguintes informações: grupo de produtos disponível para a venda, estratégia de divulgação dos produtos, plataforma de venda dos produtos, meio de pagamento utilizado e estratégia de entrega. Deve-se ressaltar que vários dos negócios investigados possuíam mais de uma opção de canal de marketing, negociação, pagamento e/ou entrega, no entanto foi considerada apenas a principal estratégia. A análise dos dados possui enfoque quantitativo, fazendo uso de técnicas descritivas.

\section{Resultados}

Foram coletados 50 anúncios e as respectivas empresas foram contatadas. No entanto, algumas informações, sobretudo relativas ao meio de pagamento, não foram disponibilizadas. Apenas foram consideradas empresas que passaram a oferecer venda à distância durante o período de distanciamento social. Os estabelecimentos foram classificados em três segmentos para melhor compreensão das estratégias de negócios, a saber: produtos básicos $46 \%$ (mercados, açougues, padarias e hortifruti); produtos prontos, lanches e bebidas $38 \%$ (sorvetes, doces, cafés, lanches, bebidas alcoólicas e petiscos) e outros produtos e serviços $16 \%$ (petshop, assistência técnica, dentre outros).

No que diz respeito a estratégia de negócios desenvolvida, foram analisadas as seguintes características do modelo de negócio emergente em resposta à pandemia: I - Estratégia de divulgação: a

DOI: $10.25286 /$ repa.v5i4.1455 
maneira pela qual a empresa apresenta seu mix de produtos aos clientes. II - Plataforma de vendas: interface que possibilita a seleção dos produtos, troca de informações sobre os produtos e fechamento do pedido. III - Plataforma de pagamentos: meio disponibilizado para pagamento dos produtos recebidos; e IV - Estratégia de entrega: canais alternativos para a chegada dos produtos até os clientes finais, devido às restrições de movimentação de pessoas e de funcionamento de muitos estabelecimentos durante a pandemia.

Em relação a estratégia de marketing $(n=50)$, há um predomínio de divulgação através da rede social Instagram em todos os segmentos, com maior percentual nos alimentos prontos e bebidas (100\%) e menor nos produtos básicos $(43,48 \%)$, no qual existe participação significativa da rede social Facebook. Tal resultado pode ser entendido pelo perfil de usuários das redes sociais consideradas. Enquanto o Instagram congrega um público mais jovem, o Facebook apresenta um perfil mais adulto dos usuários, sendo os mais utilizados na oferta de produtos básicos.

Tabela 1: Estratégia de Divulgação e Marketing

\begin{tabular}{|c|c|c|c|c|c|c|}
\hline \multirow{2}{*}{\multicolumn{2}{|c|}{$\begin{array}{l}\text { Segmentos } \\
\text { de produtos }\end{array}$}} & \multicolumn{5}{|c|}{ Estratégia de Divulgação e Marketing } \\
\hline & & \multirow{2}{*}{$\begin{array}{c}\begin{array}{c}\text { Insta- } \\
\text { gram }\end{array} \\
10\end{array}$} & \multirow{2}{*}{$\begin{array}{c}\begin{array}{c}\text { Face- } \\
\text { book }\end{array} \\
6\end{array}$} & \multirow{2}{*}{$\frac{\begin{array}{c}\text { Boca a } \\
\text { boca }\end{array}}{5}$} & \multirow{2}{*}{$\begin{array}{c}\begin{array}{c}\text { Cartaz } \\
\text { em Loja }\end{array} \\
2\end{array}$} & \multirow{2}{*}{$\frac{\text { Site }}{0}$} \\
\hline Produtos & $\mathrm{n}$ & & & & & \\
\hline básicos & $\%$ & $43,5 \%$ & $26,1 \%$ & $21,7 \%$ & $8,7 \%$ & $0,0 \%$ \\
\hline \multirow{2}{*}{$\begin{array}{c}\text { Alimentos } \\
\text { prontos/ } \\
\text { bebidas }\end{array}$} & $\mathrm{n}$ & 19 & 0 & 0 & 0 & 0 \\
\hline & $\%$ & $100,0 \%$ & $0,0 \%$ & $0,0 \%$ & $0,0 \%$ & $0,0 \%$ \\
\hline \multirow{2}{*}{$\begin{array}{c}\text { Outros } \\
\text { produtos/ } \\
\text { serviços }\end{array}$} & $\mathrm{n}$ & 5 & 1 & 1 & 0 & 1 \\
\hline & $\%$ & $62,5 \%$ & $12,5 \%$ & $12,5 \%$ & $0,0 \%$ & $12,5 \%$ \\
\hline \multirow{2}{*}{ Total } & $\mathrm{n}$ & 34 & 7 & 6 & 2 & 1 \\
\hline & $\%$ & $68,0 \%$ & $14,0 \%$ & $12,0 \%$ & $4,0 \%$ & $2,0 \%$ \\
\hline
\end{tabular}

Fonte: Autores (2020).

A forma de pagamento preferida segundo 0 levantamento $(n=39)$ é o pagamento através de máquina de cartão de crédito ou débito $(76,92 \%)$, seguida por aplicativo de pagamento $(15,38 \%)$ e transferência bancária (7,69\%). No entanto, tal opção não é uniforme nos diferentes segmentos de produtos, no grupo de alimentos prontos e bebidas ocorre a importante participação de aplicativos de pagamento $(38,46 \%)$. O que pode ser resultante da popularidade do uso de aplicativos para a aquisição de alimentos prontos no país, de forma, que os negócios tradicionais ao digitalizar seu processo optam por uma alternativa já consolidada.

Tabela 2: Estratégia de negociação e venda

\begin{tabular}{c|c|c|c|c|c|c}
\hline \multirow{2}{*}{$\begin{array}{c}\text { Segmentos de } \\
\text { produtos }\end{array}$} & \multicolumn{5}{|c}{ Plataforma de venda } \\
\cline { 2 - 7 } & $\begin{array}{c}\text { Insta- } \\
\text { gram }\end{array}$ & $\begin{array}{c}\text { Aplica- } \\
\text { tivo }\end{array}$ & Site & Telefone & Whatsapp \\
\hline \multirow{2}{*}{$\begin{array}{c}\text { Produtos } \\
\text { básicos }\end{array}$} & $\mathrm{n}$ & 0 & 1 & 1 & 6 & 15 \\
\cline { 2 - 7 } & $\%$ & $0,0 \%$ & $4,4 \%$ & $4,4 \%$ & $26,1 \%$ & $65,2 \%$ \\
\hline $\begin{array}{c}\text { Alimentos } \\
\text { prontos/ } \\
\text { bebidas }\end{array}$ & $\mathrm{n}$ & 2 & 1 & 2 & 0 & 14 \\
\cline { 2 - 7 } & $\%$ & $10,5 \%$ & $5,3 \%$ & $10,5 \%$ & $0,0 \%$ & $73,7 \%$ \\
\hline $\begin{array}{c}\text { Outros } \\
\text { produtos/ } \\
\text { serviços }\end{array}$ & $\mathrm{n}$ & 0 & 0 & 1 & 1 & 5 \\
\cline { 2 - 7 } & $\%$ & $0,0 \%$ & $0,0 \%$ & $14,3 \%$ & $14,3 \%$ & $71,4 \%$ \\
\hline \multirow{2}{*}{\begin{tabular}{c} 
Total \\
\cline { 2 - 7 }
\end{tabular}} & $\mathrm{n}$ & 2 & 2 & 4 & 7 & 34 \\
\cline { 2 - 7 } & $\%$ & $4,1 \%$ & $4,1 \%$ & $8,2 \%$ & $14,3 \%$ & $69,4 \%$
\end{tabular}

Fonte: Autores (2020).

No que concerne a plataforma utilizada para a negociação entre clientes e vendedores $(n=49)$, a pesquisa identificou o predomínio do aplicativo de troca de mensagens WhatsApp $(68,75 \%)$, seguido pelo uso do telefone $(14,58 \%)$. O predomínio das duas modalidades pode ser observado nos produtos básicos e outros produtos/serviços. Nos alimentos prontos e bebidas por sua vez, ocorre uma participação de redes sociais e sites em detrimento do telefone.

Tabela 3: Estratégia de pagamento

\begin{tabular}{c|c|c|c|c}
\hline \multirow{2}{*}{$\begin{array}{l}\text { Segmentos de } \\
\text { produtos }\end{array}$} & \multicolumn{3}{|c}{ Estratégia de pagamento } \\
\cline { 2 - 5 } & $\begin{array}{c}\text { Máquina de } \\
\text { cartão }\end{array}$ & $\begin{array}{c}\text { Aplicativo de } \\
\text { pagamento }\end{array}$ & $\begin{array}{c}\text { Transferência } \\
\text { bancária }\end{array}$ \\
\hline \multirow{2}{*}{$\begin{array}{c}\text { Produtos } \\
\text { básicos }\end{array}$} & $\mathrm{n}$ & 19 & 1 & 1 \\
\cline { 2 - 5 } & $\%$ & $90,5 \%$ & $4,8 \%$ & $4,8 \%$ \\
\hline $\begin{array}{c}\text { Alimentos } \\
\text { prontos / } \\
\text { bebidas }\end{array}$ & $\mathrm{n}$ & 6 & 5 & 2 \\
\cline { 2 - 5 } $\begin{array}{c}\text { Outros } \\
\text { produtos / } \\
\text { serviços }\end{array}$ & $\mathrm{n}$ & $46,2 \%$ & $38,5 \%$ & $15,4 \%$ \\
\cline { 2 - 5 } & $\%$ & $100,0 \%$ & $0,0 \%$ & $0,0 \%$ \\
\hline \multirow{2}{*}{ Total } & $\mathrm{n}$ & 30 & 6 & 3 \\
\cline { 2 - 5 } & $\%$ & $76,9 \%$ & $15,4 \%$ & $7,7 \%$
\end{tabular}

Fonte: Autores (2020).

Sobre a entrega dos produtos $(n=45)$, percebe-se o predomínio da entrega por motociclistas e ciclistas de aplicativos $(55,56 \%)$, seguido pela entrega do proprietário $(24,22 \%)$, funcionários do estabelecimento $(11,11 \%)$ e retirada na loja e motoristas de aplicativos, ambos com $4,4 \%$. Os produtos básicos são os únicos que apresentaram entregas por motoristas de aplicativos dado o volume 
das compras de supermercados. Alimentos prontos/bebidas e outros produtos/serviços apresentaram importante participação da entrega pelo proprietário em decorrência do menor porte de tais estabelecimentos.

Tabela 4: Estratégia de Entrega

\begin{tabular}{|c|c|c|c|c|c|c|}
\hline \multirow{2}{*}{\multicolumn{2}{|c|}{$\begin{array}{l}\text { Segmentos } \\
\text { de produtos }\end{array}$}} & \multicolumn{5}{|c|}{ Estratégia de Entrega } \\
\hline & & \multirow{2}{*}{$\begin{array}{c}\begin{array}{c}\text { Motos e } \\
\text { bicicletas } \\
\text { (app) }\end{array} \\
11\end{array}$} & \multirow{2}{*}{$\begin{array}{c}\begin{array}{c}\text { Proprie- } \\
\text { tário }\end{array} \\
3\end{array}$} & \multirow{2}{*}{$\begin{array}{c}\begin{array}{c}\text { Funcio- } \\
\text { nários }\end{array} \\
3\end{array}$} & \multirow{2}{*}{$\begin{array}{c}\begin{array}{c}\text { Retirada } \\
\text { na loja }\end{array} \\
0\end{array}$} & \multirow{2}{*}{$\begin{array}{c}\begin{array}{c}\text { Motorista } \\
\text { de app }\end{array} \\
2\end{array}$} \\
\hline Produtos & $\mathrm{n}$ & & & & & \\
\hline & $\%$ & $57,9 \%$ & $15,8 \%$ & $15,8 \%$ & $0,0 \%$ & $10,5 \%$ \\
\hline \multirow{2}{*}{$\begin{array}{c}\text { Alimentos } \\
\text { prontos/ } \\
\text { bebidas }\end{array}$} & $\mathrm{n}$ & 10 & 6 & 1 & 2 & 0 \\
\hline & $\%$ & $52,6 \%$ & $31,6 \%$ & $5,3 \%$ & $10,5 \%$ & $0,0 \%$ \\
\hline \multirow{2}{*}{$\begin{array}{l}\text { Outros } \\
\text { produtos } \\
\text { /serviços }\end{array}$} & $\mathrm{n}$ & 4 & 2 & 1 & 0 & 0 \\
\hline & $\%$ & $57,1 \%$ & $28,6 \%$ & $14,3 \%$ & $0,0 \%$ & $0,0 \%$ \\
\hline \multirow{2}{*}{ Total } & $n$ & 25 & 11 & 5 & 2 & 2 \\
\hline & $\%$ & $55,6 \%$ & $24,4 \%$ & $11,1 \%$ & $4,4 \%$ & $4,4 \%$ \\
\hline
\end{tabular}

Fonte: Autores

Por fim, foi aplicado um teste Qui-quadrado (x2) para cada cross table desenvolvida nesta pesquisa. 0 teste Qui-quadrado faz uma comparação entre frequências de amostras independentes, permitindo avaliar a relação entre as variáveis, e identificar diferenças estatisticamente significativas entre 0 resultado esperado e o observado. Após a aplicação do teste foram observados os seguintes $p$-valores: estratégia de marketing 0,02 , estratégia de vendas 0,174 , estratégia de pagamentos 0,024 , e estratégia de entrega 0,217 .

\section{Conclusões}

Esta pesquisa contribui para o entendimento deste fenômeno ao fornecer um panorama das mudanças forçadas que têm ocorrido nos pequenos negócios brasileiros durante a pandemia. Ao mesmo tempo, aponta indícios para o desenvolvimento de estudos mais abrangentes e políticas públicas de apoio a este importante grupo de organizações. Percebe-se, desta forma, que a combinação de divulgação através de mídias sociais (Instagram), com o uso de aplicativos de troca de mensagens (WhatsApp) para seleção de produtos e fechamento das compras, pagamento através de máquinas de cartão de crédito que são levadas até o cliente através de entregadores (cadastrados em aplicativos de entrega), configuram o modelo de negócio utilizado por empresas na região do Recife, Brasil. Tal modelo de negócio tem conseguido atender as necessidades de abastecimento da população em meio a pandemia do COVID-19, auxiliando nas ações sanitárias de isolamento social. As estratégias desenvolvidas em cada segmento analisado seguem esta lógica, com pequenas variações decorrentes do público predominante de cada segmento.

Como indicado por Okano, Simões e Langhi [20], Rogers [21] e Schallmo, Williams e Boardman [22], a transformação digital exige o desenvolvimento de um novo modelo de negócio, e isto só é possível graças a mudanças nos hábitos do consumidor $\mathrm{e}$ transformações na sociedade. Um recurso que viabiliza os negócios digitais é a popularização do smartphone no país. Segundo levantamento da FGVSP [33], em 2019 havia 230 milhões de celulares ativos. A ampliação no acesso a smartphones e conexão de banda larga possibilitaram outra importante mudança de hábito da sociedade que é o acesso a redes sociais. De acordo com o relatório Digital in Brazil [34], o brasileiro passa em média três horas e trinta e quatro minutos online em redes sociais, as mais populares no país são Youtube, Facebook, WhatsApp e Instagram, enquanto as preferidas, segundo o mesmo relatório, são Instagram e Facebook.

Outra transformação na sociedade é a possibilidade da troca de mensagens instantâneas e de baixo custo através de aplicativos de troca de mensagens. Tais ferramentas têm possibilitado a troca de informações para os mais variados fins, dentre eles as atividades comerciais. Segundo informações do Global Messaging Apps [35], o Brasil em 2019 tinha 120 milhões de usuários de WhatsApp. Várias grandes empresas já desenvolveram canais de atendimento ao cliente através de aplicativos de trocas de mensagens e a pandemia tem levado os empreendedores individuais, microempresas e empresas de pequeno porte a buscarem também tal alternativa.

Esta ampliação nas possibilidades dos novos modelos de negócios não seria possível sem o desenvolvimento de novas formas de pagamento nas últimas décadas. Nesse sentido, destaca-se que o dinheiro eletrônico tem substituído o papel-moeda e está a cada dia mais presente na vida das famílias. A popularização do uso de máquinas de cartão de crédito faz parte deste processo de modificação de hábitos da sociedade. As máquinas de cartão já

DOI: $10.25286 /$ repa.v5i4.1455 
estavam presentes em $46 \%$ dos pequenos negócios brasileiros em 2018, enquanto no setor de varejo, tal patamar é de $68 \%$, de acordo com levantamento do SEBRAE [36].

Por fim, por mais possibilidades de divulgação, negociação e pagamentos existentes, sem um mecanismo que garanta a chegada dos produtos aos clientes finais, o ciclo do produto não será finalizado, como destacam Gevaers et al. [25], Alenn et al. [26] e Janjevic e Winkenback [27]. O problema da última milha tem sido resolvido com a disseminação de aplicativos de entregas de produtos direcionados a priori para entrega de refeições e lanches (Ifood, Uber Eats, Rappi, James). De acordo com o IBGE [37] em 2019, havia 13,7 milhões trabalhadores atuando como motoristas de aplicativos e entregas. Os entregadores através de motocicletas e bicicletas têm circulado pelas ruas do país garantindo a entrega de produtos e abastecimento durante a pandemia, de forma a contribuir para a manutenção do isolamento social.

O estudo possui como limitações o tamanho da amostra e a dificuldade de obtenção das mesmas em período de pandemia, período em que muito custoso para os gestores a paralisação de suas atividades para $o$ atendimento das demandas dos pesquisadores. No entanto, tal estudo mostra indícios de um movimento de digitalização que está ocorrendo forçadamente e improvisadamente no contexto da pandemia do COVID-19, e mostra as estratégias de pequenos negócios para se adaptar e sobreviver. Considera-se que esta pesquisa pode contribuir para 0 desenvolvimento de um framework que auxilie microempreendedores individuais, microempresas e empresas de pequeno porte no processo de digitalização de suas atividades.

\section{Referências}

[1] OLIVEIRA, Wanderson Kleber de; DUARTE, Elisete; FRANCA, Giovanny Vinícius Araújo de, GARCIA, Leila Posenato. Como o Brasil pode deter a COVID-19. Epidemiol. Serv. Saúde, Brasília, v. 29, n. 2, 2020. Disponível em: $<$ http://www.scielo.br/scielo.php?script=sci_arttext\& pid =S2237-96222020000200200\&lng =en\&nrm=iso > Acesso em: 03 jul. 2020.

[2] WORLD HEALTH ORGANIZATION WHO. China shows COVID-19 responses must be tailored to the local context". Disponível em: http://www.euro.who.int/en/health-topics/healthemergencies/coronavirus-covid19/news/news/2020/4/china-shows-covid-19- responses-must-be-tailored-to-the-local-context> Acesso em: 08 jul. 2020.

[3] WORLD HEALTH ORGANIZATION WHO. Advice on the use of masks in the context of COVID-19. Disponível em <https://apps.who.int/iris/bitstream/handle/10 665/332293/WHO-2019-nCov-IPC_Masks2020.4-eng.pdf> Acesso em: 08 jul. 2020.

[4] INTERNATIONAL MONETARY FUND IMF. World Economic Outlook, April 2020: The Great Lockdown. $2020 . \quad$ Disponível em: $<$ https://www.imf.org/en/Publications/WEO/Issues/2 020/04/14/weo-april-2020>. Acesso em: 17 abr. 2020.

[5] BANCO CENTRAL DO BRASIL BACEN. Economic activity report. 2019 Disponível em: <https://www.bcb.gov.br/en>. Acesso em: 24 abr. 2020.

[6] FUNDAÇÃO GETÚLIO VARGAS FGV-SP. Pesquisa Anual do Uso de TI. 2019. Disponível em: <https://eaesp.fgv.br/ensinoeconhecimento/centros/ cia/pesquisa>. Acesso em: 17 abr. 2020.

[7] ESTADOS UNIDOS DA AMÉRICA. US. Government Response to Coronavirus, COVID-19, 2020. Disponível em: < https://www.usa.gov/coronavirus>. Acesso em: 17 abr. 2020.

[8] EUROPEAN COMISSION. COVID-19: Commission sets out European coordinated response to counter the economic impact of the Coronavirus. 2020. Disponível em: <https://ec.europa.eu/commission/presscorner/deta il/en/ip_20_459>. Acesso em: 17 abr. 2020.

[9] BRASIL. Medidas Econômicas de Combate à Covid-19. 2020. Disponível em: <https://www.gov.br/economia/pt-br/centrais-deconteudo/apresentacoes/2020/2020-04-15-medidasenfrentamento-da-crise-1530hs.pdf > Acesso em: 30 abr. 2020.

[10] CNN. More than 600,000 small businesses closed their doors with coronavirus. 2020. Disponível em:

<https://www.cnnbrasil.com.br/business/2020/04/0 9/mais-de-600-mil-pequenas-empresas-fecharamas-portas-com-coronavirus> Acesso em 29 abr. 2020.

[11] BRASIL. Medida Provisória nº 936, de $1^{\circ}$ de abril de 2020.2 Disponível em: <http://www.planalto.gov.br/ccivil_03/_ato20192022/2020/mpv/mpv936.htm>. Acesso em: 30 abr. 2020. 
[12] BRASIL. Lei Complementar 123, de 14 de dezembro de 2006. Institui o Estatuto Nacional da Microempresa e da Empresa de Pequeno Porte. Diário Oficial da União. Brasília, 2006. Disponível em: $<$ http://www.planalto.gov.br/ccivil 03/leis/lcp/lcp12 3.htm>. Acesso em: 17 abr. 2020.

[13] BRASIL. Lei Complementar 128, de 19 de dezembro de 2008. Altera a Lei Complementar no 123, de 14 de dezembro de 2006. Diário Oficial da União. Brasília, 2008. Disponível em: <http://www.planalto.gov.br/ccivil_03/leis/lcp/lcp12 8.htm>. Acesso em: 17 abr. 2020.

[14] BRASIL. Lei Complementar 128, de 19 de dezembro de 2008. Altera a Lei Complementar no 123, de 14 de dezembro de 2006. Diário Oficial da União. Brasília, 2008. Disponível em: $<$ http://www.planalto.gov.br/ccivil_03/leis/lcp/lcp15 5.htm> Acesso em: 17 abr 2020

[15] GLOBAL ENTREPREUNESHIP MONITOR GEM 2018/2019 Global Report. 2019. Disponível em: <https://www.gemconsortium.org/file/open?fileI $d=50213>$. Acesso em: 17 abr. 2020.

[16] SERVIÇO BRASILEIRO DE APOIO A MICRO E PEQUENAS EMPRESAS SEBRAE. Sobrevivência e mortalidade de empresas, 2020. Disponível em: <https://m.sebrae.com.br/Sebrae/Portal\%20Sebrae /Ufs/SP/Pesquisas/CAUSA\%20MORTIS_vf.pdf> Acesso em: 30 abr. 2020.

[17] SERVIÇO BRASILEIRO DE APOIO A MICRO E PEQUENAS EMPRESAS SEBRAE. O Impacto da pandemia de coronavírus nos Pequenos Negócios, 2nd ed. $2020 . \quad$ Disponível em: <https://www.sebrae.com.br/sites/PortalSebrae/arti gos/impactos-e-tendencias-da-covid-19-nospequenos-

negocios,5e8fbd0c7d711710VgnVCM1000004c00210 aRCRD>. Acesso em: 30 abr. 2020.

[18] GOUVEIA DE SOUSA GS \& NPD. Tendências em Varejo e Foodservice. 2019. Disponível em: <https://www.gcca.org/sites/default/files/3\%20-

\%20Food\%20Trends.pdf >. Acesso em: 07 abr. 2020.

[19] EBIT-NIELSEN. Webshoppers - 40a ed. 2019. Disponível em: <https://www.ebit.com.br/webshoppers\#ws-free>. Acesso em: 12 Maio 2020.

[20] OKANO, M.T, SIMÕES, E.A, LANGHI, C. Plataformas de negócios digitais: o poder da transformação digital nos dispositivos móveis. Research, Society and Development, v. 9, n. 2. 2019. Disponível

em:
$<$ https://rsd.unifei.edu.br/index.php/rsd/article/view /2097>. Acesso em: 09 maio 2020.

[21] ROGERS, D. L. Transformação Digital: Repensando o seu negócio para a era digital. São Paulo, Autêntica Business, 2018.

[22] SCHALLMO, D, WILLIAMS, C. A AND BOARDMAN, L. Digital Transformation Of Business Models Best Practice, Enablers, And Roadmap'. International Journal of Innovation Management, 21(8). 2017. Disponível em: <https://doi.org/10.1142/S136391961740014X>. Acesso em: 07 abr. 2020.

[23] DIOGO, Ricardo Alexandre; JUNIOR, Armando Kolbe; SANTOS, Neri. A transformação digital e a gestão do conhecimento: contribuições para a melhoria dos processos produtivos e organizacionais. P2p E Inovação, v. 5, n. 2, p. 154-175, 2019.

[24] EXAME. O fenômeno segue: Magalu dobra ecommerce e lucro sobe 13\%. 2019. Disponível em: <https://exame.abril.com.br/negocios/magazineluiza-tem-lucro-ajustado-127-maior-no-3otrimestre >. Acesso em: 09 Maio 2020.

[25] GEVAERS, R., VAN DE VOORDE, E. e VANELSLANDER, T. Characteristics and typology of lastmile logistics from an innovation perspective in an Urban context. City Distrib. Urban Freight Transp.: Multiple Perspect. 2011. Disponível em: <https://doi.org/10.4337/9780857932754.00009>. Acesso em: 17 abr. 2020.

[26] ALLEN, J., PIECYK, M., PIOTROWSKA, M., MCLEOD, F., CHERRETT, T., GHALI, K., NGUYEN, T., BEKTAS, T., BATES, O., FRIDAY., A, WISE, S AND AUSTWICK, M. Understanding the impact of $e-$ commerce on last-mile light goods vehicle activity in urban areas: the case of London', Transportation Research Part D: Transport and Environment. 2017. Vol. 61 No. 1, pp. 325-338.

[27] JANJEVIC, M. e WINKENBACH, M. Characterizing urban last-mile distribution strategies in mature and emerging e-commerce markets' Transportation Research Part A: Policy and Practice. 2020. Vol. 133 pp. 164-196.

[28] BLANCO, E., FRANSOO, J. Reaching 50 million Nanostores: Retail Distribution in Emerging Megacities. 2013. Technische Universiteit Eindhoven, Eindhoven, The Netherlands

[29] Zuo, T, Wei, H, Chen, N and Zhang, C. (2020) 'First-and-last mile solution via bicycling to improving transit accessibility and advancing transportation equity'. Cities, 2020. Disponível em: 
<https://doi.org/10.1016/j.cities.2020.102614>. Acesso em: 07 abr. 2020.

[30] KAPSER, S e ABDELRAHMAN, M. 'Acceptance of autonomous delivery vehicles for last-mile delivery in Germany - Extending UTAUT2 with risk perceptions'. Transportation Research Part C: Emerging Technologies. 2020. Vol. 111 pp. 210 - 225.

[31] FERREIRA, Líndice Thiengo. Transformação digital: aplicações e limitações de seu uso em empresas de seguro no Brasil. 2018. Tese de Doutorado.

[32] GUIMARÃES JR, D,S, SANT'ANNA, $C, H, M$, SOARES, E,J,O, MEDEIROS, D,D, MELO, F,J,C. Measurement of logístics service quality of $\mathrm{e}$ commerce. International Journal of Logistics Systems and Management. 2020. Disponível em: 10.1504/IJLSM.2020.10029096. Acesso em: 12 abr. 2020

[33] FUNDAÇÃO GETÚLIO VARGAS FGV-SP. Annual IT Use Survey. 2019. Disponível em: <https://eaesp.fgv.br/ensinoeconhecimento/centros/ cia/pesquisa >. Acesso em: 17 abr. 2020.

[34] DIGITAL IN BRAZIL. 2019 Disponível em: <https://datareportal.com/reports/digital-2019brazil>. Acesso em: 17 abr. 2020.

[35] GLOBAL MESSAGING APPS. 2019. Disponível em: <https://www.emarketer.com/content/globalmessaging-apps-2019\#page-chart>. Acesso em: 17 abr. 2020.

[36] SERVIÇO BRASILEIRO DE APOIO A MICRO E PEQUENAS EMPRESAS SEBRAE. DataSebrae Maquininha de cartão. 2019. Disponível em: $<$ https://datasebrae.com.br/maquininha-de-

cartao/\# percentualtotal>. Acesso em: 30 abr. 2020.

[37] INSTITUTO BRASILEIRO DE GEOGRAFIA E ESTATÍSTICA IBGE. PNAD Contínua. 2019. Disponível em:

<https://www.ibge.gov.br/estatisticas/multidominio/ condicoes-de-vida-desigualdade-e-pobreza/17270pnad-continua. html? $=\& \mathrm{t}=0$-que-e. $>$. Acesso em: 30 abr. 2020. 